\title{
CACERÍA DEL PECARÍ DE COLLAR (Pecarí tajacu) (ARTIODACTYLA: TAYASSUIDAE) EN TZUCACAB, YUCATÁN, MÉXICO
}

\section{MARCOS ALBERTO BRICEÑO MENDEZ ${ }^{1}$, RUBEN MONTES PEREZ, WILIAN AGUILAR CORDERO Y ADDY POOL CRUZ ${ }^{2}$}

\author{
${ }^{1}$ Campus de Ciencias Biológicas y Agropecuarias, Facultad de Medicina Veterinaria y \\ Zootecnia, Universidad Autónoma de Yucatán, Carretera Mérida-Xmatkuil km 15.5, s/n, \\ Mérida, Yucatán, Fax: 99994232 05- ext. 32
}

${ }^{2}$ Instituto Tecnologico de Conkal km 16.3 ant. carr. Mérida-Motul C. P. 97345 Conkal, Yucatán Tel( S) (999) 124130 Fax (999) 124131.

correo electrónico: marc12_87@hotmail.com

\begin{abstract}
Resumen: La utilización de la fauna nativa en la dieta de la población rural es una práctica común en muchas comunidades de la República Mexicana. El objetivo de este estudio fue describir el uso extractivo de los pecaríes de collar en el municipio de Tzucacab, Yucatán. La recolección de datos incluyó visitas a los sitios de caza y 30 entrevistas semi-estructuradas aplicadas a cazadores. Se registraron en total 93 eventos de caza, en los cuales se cazaron 22 pecaríes (13 machos y 9 hembras) con una biomasa total de 374 kg y se registraron 17 pecaríes heridos, este es el primer registro de los animales heridos, pero no aprovechados para el municipio. No se encontraron diferencias significativas en la preferencia de caza por sexos. De los 30 cazadores, el $32 \%$ sale diario, el 28\% cada semana y un $40 \%$ cada mes. Se diferenciaron tres técnicas de caza (batida, espía y lampareo). La cacería de pecarí de collar esta asociada con aspectos culturales y sociales y es una actividad que otorga beneficios económicos a los campesinos cazadores mas allá de proporcionar alimento. La batida es la técnica más utilizada con fines de subsistencia y con la que se caza el mayor número de animales, pero esta tiene un efecto perjudicial sobre la población de $P$. tajacu en el área de estudio, debido a que al utilizar esta técnica las probabilidades de éxito de caza son mayores comparada con la espía y el acecho. Deben evaluarse alternativas de extracción sustentables para pecaríes. Mediante talleres de acción participativa se plantearon propuestas por los pobladores para que se realice un aprovechamiento sustentable de este recurso, establecer unidades de manejo y aprovechamiento en su modalidad intensiva (UMA) sería una alternativa para el manejo sustentable del recurso en el municipio.
\end{abstract}

Palabras clave: Cacería, Pecari tajacu, heridos, batida, espía, lampareo.

Abstract: The use of native fauna in the diet of rural people is a common practice in many
communities of the Mexican republic. The aim of this study was to describe the extractive use
of collared peccaries in a municipality named Tzucacab in the Mexican state of Yucatan. The
data collection included site visits and semi-structured interviews with 30 hunters. A total of Revista Mexicana de Mastozoología. Nueva Época Año 1, No. 1:8-18. 2011. 
93 hunting events in which peccary hunted 22 (13 males and 9 females) with a total biomass of $374 \mathrm{~kg}$ and 17 peccary were only injured this is one of the first studies that record animals wounded but not killed. $\mathrm{X}^{2}$ test indicates no evidence of hunting preference by gender (2.3). Of the 30 hunters who practice hunting, 32\% perform daily trips while $28 \%$ and $40 \%$ once per week and per month respectively. The collared peccary hunt, which is associated with cultural and social issues, is an activity that provides economic benefits to hunting farmers beyond providing food. The "batida" (when some hunters push animals toward a site where other are hiding) is the traditional hunting technique, but this has a detrimental effect on the population of P. tajacu in the area of study, divide to use this technique to the chances of success are over game versus the "spy" and the techniques are "lampareo" practiced in Tzucacab, so alternatives should be evaluated for sustainable extraction peccaries. Through workshops participatory action by the residents to put forward proposals to be made sustainable utilization of this resource, establish management and development units in intensive mode (UMA) is an alternative for sustainable resource management in the municipality.

Key words: Hunting, Pecari tajacu, wounded, "Batida", "Spy", "lampareo".

\section{INTRODUCCIÓN}

La cacería de subsistencia es una práctica común en las comunidades rurales de México así como en el trópico cuyo propósito es principalmente de autoconsumo, y para la convivencia entre los hombres y la socialización de los jóvenes, donde "gusto y necesidad" son las dos ideas básicas de cazar (Montiel et al., 1999). En tiempos recientes y debido a cambios socioculturales, la incorporación de armas de fuego y la necesidad de satisfacer carencias como la alimentación, la cacería se ha incrementado y la presión sobre la fauna silvestre es mayor, al grado tal que se ha documentado la disminución o extinción local de algunas especies de caza importantes (March, 1995).

En México la cacería se ha practicado desde tiempos prehispánicos. Esta actividad forma parte de las ceremonias tradicionales y del folklore de los pueblos indígenas y campesinos de los diferentes estados de la república (Montiel et al., 1999). La fauna silvestre ofrece numerosas especies de uso potencial y es una fuente alternativa de proteínas para las poblaciones rurales. Por esta razón, debe de considerarse como un recurso del cual se puede obtener grandes beneficios. La búsqueda de un aprovechamiento sustentable de los recursos naturales requiere el entendimiento de las interacciones entre naturaleza y sociedad, así como la combinación de diferentes actores sociales, trabajar en conjunto, aun cuando la información sea incierta y limitada (Kates, 2001). Así mismo, se necesita brindar oportunidades viables de subsistencia a los habitantes de comunidades rurales, ya que cualquier discurso conservacionista será desechado si ellos no tienen los recursos para cubrir sus necesidades básicas (Montiel et al., 1999). La cacería se ha 
desarrollado como una actividad del hombre para obtener beneficios de la fauna silvestre. Actualmente este aprovechamiento se lleva a cabo principalmente por distintos grupos del continente africano, indígenas y campesinos de las zonas tropicales de américa latina. Diversos estudios han demostrado que la fauna silvestre es un recurso ampliamente usado y que en general carece de regulación y reglamentación, lo que constituye una amenaza para el mantenimiento de las especies de caza (Redford y Robinson, 1997; Vázquez et al., 2006). Actualmente se ha analizado la forma en que esta actividad pueda ser sustentable, considerando las tradiciones culturales, las especies que se utilizan, la importancia económica de ellas, el tamaño de las poblaciones y la manera adecuada de administrar la fauna silvestre (Montiel et al., 1999).

En México diversos investigadores han abordado con profundidad el tema de la cacería, pero la mayoría de los trabajos se centran en el impacto que tiene sobre todas las especies de un sitio o región y no se hace énfasis en una sola especie en particular (Naranjo et al., 2004) En la Península de Yucatán investigadores han realizados estudios centrados en áreas de conservación (Escamilla et al., 2000). Y pocos abordan el tema en áreas con asentamientos humanos (Reyna-Hurtado y Tanner, 2007). Se conoce poco de cómo los habitantes rurales utilizan la fauna silvestre y que factores culturales y biológicos, influyen en la cacería de subsistencia.
Debido a esto, es necesario analizar y evaluar el impacto de la cacería sobre la fauna silvestre, este estudio describe el impacto que tiene la cacería exclusivamente sobre el pecarí de collar y se reporta por vez primera datos de pecaríes heridos, pero no aprovechados en el municipio de Tzucacab, Yucatán.

\section{ÁREADEESTUDIO}

El estudio se realizó en el municipio de Tzucacab, localizado al sur del Estado de Yucatán, a una distancia de $111 \mathrm{~km}$ de la ciudad de Mérida. Limita al norte con los municipios Tixmehuac, Chacsinkin, al sur con el Estado de Quintana Roo, al este con el municipio de Peto y al oeste con el municipio de Tekax; tiene una superficie de $1,289 \mathrm{~km}^{2} \mathrm{y}$ su altura promedio es de 36 msnm (Figura 1; Municipios de México, 2002). El clima es de tipo $A w_{1}(x)$ (i) g que es cálido subhúmedo intermedio con lluvias en verano. La temperatura media anual es de $25.8^{\circ} \mathrm{C}$ y la precipitación media anual alcanza los $1061.2 \mathrm{~mm}$ (CNA, 2005).

Los tipos de vegetación predominantes en el área son selva baja caducifolia, selva baja inundable, selva mediana subcaducifolia además existen zonas de acahuales y áreas de uso agropecuario. (Flores y Espejel, 1994).

Se presentan los suelos del tipo litosol (Tsekel), rendzina ( $\mathrm{K}^{\prime}$ ankab) y luvisolcrómico de textura fina y sin fases, dirigidos hacia el norte y este respectivamente, con textura media. (Duch, 1988). De la extensión total territorial que ocupada el municipio, para la agricultura se tiene $1.77 \%$, donde se 


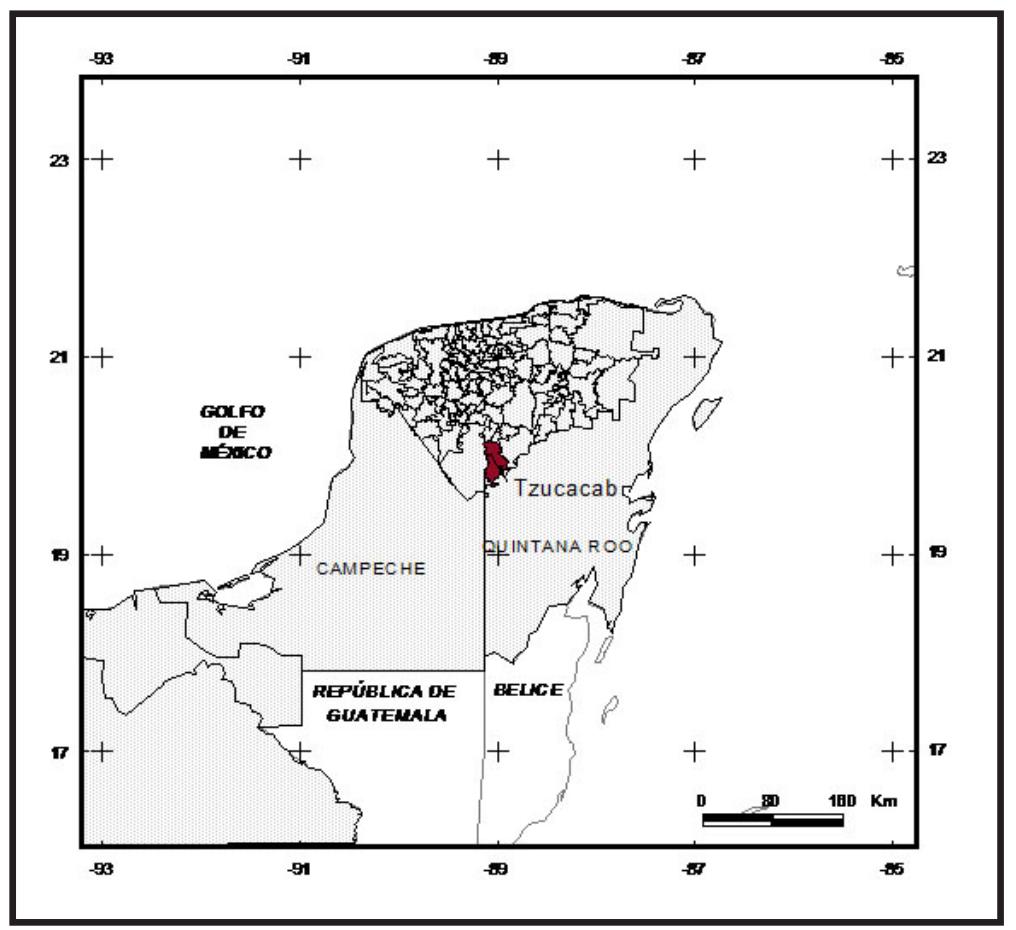

Figura 1. Ubicación del Municipio de Tzucacab en el estado de Yucatán.

cultivan maíz, papaya maradol, melón, calabaza, chile y sandia, parapastizal 18.41\%.(INEGI, 2010).

Las actividades de sustento se desarrollan principalmente en la milpa, en el solar o huerto familiar y el monte. El solar o huertos familiares son comunes en la zona y en ellos se cultivan los frutales, hortalizas, plantas medicinales y deornato; se crían animales como las gallinas, pavos y cerdos. En la selva se lleva a cabo la extracción forestal de productos maderables y no maderables como la colecta de hojas de palma de
Guano y la cacería, (Teran y Rasmussen, 1992).

Todos los ejidos que conforman el municipio de Tzucacab están comunicados con carreteras pavimentadas. Cuentan con servicio de electricidad, agua potable con toma domiciliaria, teléfono satelital, además cuentan con escuelas bilingües hasta el nivel primarias. Las clínicas de servicios médicos rurales solo se encuentran en la cabecera municipal y en el Ejido Noh- Bec (INEGI, 2010). 


\section{MÉTODOS}

En la investigación etnobiológica se utiliza los métodos de investigación cuantitativo y cualitativo (Vela, 2001). En este estudio se usaron ambos métodos.

\section{Entrevistas}

Se utilizo la técnica de la entrevista semiestructurada $(n=30)$, que consiste en encuentros repetidos, cara a cara, entre un investigador y sus informantes, los cuales se orientan a entender las perspectivas del entrevistado sobre su vida, experiencia o situaciones personales tal y como son expresadas por sus propias palabras (Vela, 2001). Así como el conocimiento sobre aspectos biológicos de los pecaríes de collar.

\section{Encuestas}

Las encuestas fueron aplicadas generalmente en la tarde ya que a esa hora los cazadores se encuentran en sus casas después de su jornada de trabajo. Los cuestionarios y encuestas fueron aplicados a campesinos - cazadores activos, con promedio de edad de 40 años. Esta metodología es inductiva, humanista, considera escenarios y actores de manera holística, tratando siempre de comprender a los actores dentro de su propio marco de referencia (Vela, 2001).

\section{Seguimiento de la cacería}

El seguimiento y análisis de cacería se realizó durante estancias de 15 días al mes entre septiembre de 2009 a julio de 2010. Se registró el peso, sexo, talla y estado reproductivo de los pecaríes cazados. También se registró el número de animales heridos por armas de fuego pero que no fueron aprovechados por el cazador. Los indicadores de cacería fueron la frecuencia de salidas de caza (días /semana /mes) y el número de cazadores participantes, así como la técnica que utilizan. Se registró el número de individuos cazados en cada tipo de vegetación existentes en el municipio (selva baja caducifolia, selva baja inundable, selva mediana subcaducifolia, y vegetación secundaria; Flores y Espejel, 1994), así como campos de cultivos (localmente llamados milpas) y áreas ganaderas (llamadas potreros).

\section{Análisis estadístico}

Los datos de campo recabado mediante las encuestas se codificaron en una base datos diseñados en el programa Excell y para el análisis de los datos cuantitativos se aplicó el análisis estadístico descriptivo, sacando frecuencias y promedios.

\section{RESULTADOS}

Se registró un total de 93 salidas de cacería, de los 30 cazadores que practican la cacería el 32\% sale diario, 28\% cada semana y el $40 \%$ cada mes. Se diferenciaron tres técnicas de caza 
empleadas en el área, de las cuales la batida (donde un grupo de personas cubren un área grande y dirigen animales hacia cazadores escondidos en un lugar determinado) es la técnica más utilizada. En esta actividad se obtuvo un promedio de 18 participantes (Cuadro 1).

Se registró un total de 22 pecaríes de collar cazados (13 machos y 9 hembras) y 17 heridos, el impacto que tiene esta consecuencia de la actividad de la cacería representa una pérdida anual de hasta cerca del $77 \%$ del total de animales extraídos de la población y no aprovechados (1.88/2.44) animales/ comunidad/mes. Con base a los individuos capturados, no se encontró diferencia significativa entre el nivel de preferencia de caza y sexo $\left(X^{2}=2.3\right)$, los campesinos cazadores mencionan que para esta especie no realizan alguna selección y por lo general aprovechan tanto hembras como machos. El total de biomasa obtenida a través de la cacería fue de $374 \mathrm{~kg}$. Se registro el uso de 16 perros en la cacería del pecarí de collar. Solamente la técnica de la batida y la espía emplean perros de caza (Cuadro 1).

Se registró el mayor número de individuos cazados en vegetación secundaria con un total de 12 individuos cazados. La selva baja inundable presentó el menor número de individuos cazados con 2, mientras que las milpas y los potreros se aprovecharon 4 individuos en cada uno. No se registraron individuos cazados en la selva mediana subcaducifolia. Se obtuvo un peso promedio para las hembras cazadas de $17.38 \mathrm{~kg}$, para los machos fue de $16.73 \mathrm{~kg}$,
7 hembras presentaron estado reproductivo adulto, seguido de 6 machos. 7 machos fueron juveniles y sólo 2 hembras presentaron este estado de desarrollo.

A partir del análisis de los individuos cazados, no se registraron crías lo cual indica que los campesinos cazadores evitan cazar animales que no tiene la talla suficiente como para ser aprovechados para su consumo o comercialización.

Los campesinos cazadores propusieron estrategias de conservación y manejo sustentable del pecarí de collar, entre las cuales el establecer una UMA (Unidad de Manejo y Aprovechamiento Sustentable) de acuerdo con la legislación mexicana NOM-059 SEMARNAT-2010 de modalidad intensiva y extensiva se consideró como una estrategia adecuada para el municipio.

\section{DISCUSIÓN}

El pecarí de collar es apreciado por su carne y piel a lo largo de su área de distribución (desde Argentina hasta el sur de los Estados Unidos).

En las tierras bajas de Venezuela y Ecuador, el pecarí de collar ocupa el primer lugar de la escala de las especies más aprovechadas (Vickers, 1984).

En una comunidad aledaña a la Reserva de la Biosfera de los Petenes, Campeche se realizo un estudio y se encontró que el 63\% del total de carne silvestre que se consume corresponde al pecarí de collar (León, 2006). Sin embargo, existen diferencias en número de individuos cazados en otros sitios. Los 
registros de caza de Calakmul, Campeche, indican que el pecarí de collar $(n=100)$ es la segunda especie más cazada por debajo del Tepezcuintle, Cuniculus paca $(\mathrm{n}=247)$ (Jorgenson, 1993).

En este estudio, se registraron 22 pecaríes cazados en el municipio de Tzucacab, mientras que Segovia (2001) reportó solamente 17 animales cazados por año en el mismo lugar, este autor reporta que el pecarí de collar representa el $10.55 \%$ del total anual de especies aprovechadas. Esto lo ubica detrás del venado cola blanca (Odocoileus virginianus) con un $22.22 \%$, y del venado temazate gris (Mazama pandora) con un $44.4 \%$ de aprovechamiento anual.

El aumento en la presión de caza sobre el pecarí de collar se da en gran medida por las formas en que esta especie es aprovechada, y al ocupar áreas con perturbaciones humanas queda a la deriva de cualquier cazador.

Así mismo la ven como una especie perjudicial, ya que invaden sus milpas y destruyen sus cultivos principalmente maíz, calabaza y frijol, por lo tanto se resuelve el problema cazándolo. En un estudio se evaluó el daño que causan los mamíferos silvestres a los cultivos de maíz, la especie que causó más daño fue el pecarí de collar (35.26\%), seguido del mapache (39.50\%) (Gallegos et al., 2004). Redford y Robinson (1987) señalan que la fauna silvestre es importante en el contexto cultural y nutricional de acuerdo al tipo de grupo humano que la utiliza; esto es por las formas particulares de apropiación de los recursos y la manera en que la cacería se articula con las demás actividades extractivas y productivas.

Muchos de los estudios que se realizan sobre cacería solo reportan las especies de caza, el peso, el número de individuos aprovechados y patrones de caza por las comunidades (Vickers, 1984). Aquí presentamos también el número de pecaríes heridos y evaluamos el impacto que tiene esta consecuencia de la actividad de la cacería para las poblaciones de pecari. Al no encontrar diferencias significativas en la preferencia por sexo en este estudio, esto indica que los campesinos cazadores no realizan una selección en cazar hembras o machos, ya que por lo general esta especie forman grupos familiares que van de 8 a 14 individuos integrados por machos, hembras y crías, el numero de hembras puede llegar a ser mayor en una proporción de 3:1 (Martínez-Gallardo y Sánchez-Cordero, 1997). Esto contrasta con la caza de venados, una especie donde los campesinos prefieren cazar machos debido al tamaño del animal y por que consideran que al dejar a las hembras siempre habrá reproducción y consecuentemente el recurso estará siempre disponible.

El hecho de que se obtuvieran un total de 374 kg de carne de pecarí en Tzucacab confirma que es una especie cazada generalmente para consumo humano. La carne de animales silvestres es un recurso de gran importancia para la población humana rural. Para Tzucacab en el 2001 se registraron un total de $238 \mathrm{~kg}$ de biomasa animal correspondiente únicamente al pecarí de collar (Segovia, 2001).

La técnica de batida, es una actividad 
ilegal y la gente se cuida de no ser vistos por las autoridades, sobretodo ante los funcionarios de las instituciones encargadas de la regulación, lo que provoca que las actividades tradicionales de consumo y venta local de animales sean vistas como prácticas clandestinas y sin control aparente. Esta visión es contraria a la que tienen los habitantes locales, además de que existen pocas vías de diálogo y negociación para que ambas visiones sean compatibles.

La batida es el método más practicado por los cazadores del sur de Yucatán (Segovia, 2001). En el ejido de Tixcacaltuyub, Yucatán se reportaron 5 técnicas para la caza, tres son similares a lo registrado en este trabajo, los otros dos, la "sorpresa" y la "espera" que son variantes del acecho y lampareo (Mandujo y Rico-Gray, 1991). En Yaxcaba, Yucatán, se reconocen tres tipos de caza: la batida, la espía y cazar al caminar el monte (Montiel et al., 1999).

El uso de perros parece ser clave del éxito de la caza, podría tener un impacto considerable sobre la especie cazada (Jorgenson, 1993; 1995). Se analizaron los sitios de caza y tipo de vegetación donde fueron cazados los individuos reportados, y la mayor cantidad de individuos cazados se registró en vegetación secundaria. La vegetación secundaria ofrece variadas etapas sucesionales, sirve como refugio por la densidad de los arbustos y herbáceas presentes, diversas especies entre ellas el pecarí de collar se favorecen con la amplitud de efecto borde provocado por actividades como el desmonte, apertura de tierras para cultivos diversos y la milpa (Reyna-Hurtado y Tanner, 2005; Sowls, 1997).

Es necesario que dependencias estatales y municipales, así como organizaciones no gubernamentales cumplan con la función de resguardar los recursos faunísticos y lleven a cabo programas de manejo y conservación de especies que tienen un potencial elevado de reproducción y adaptación como el pecarí de collar, sobre todo en poblaciones rurales donde la necesidad de alimentación son difíciles de cubrir y por lo cual en ocasiones se hace uso irracional de los recursos disponibles, lo que provoca la vulnerabilidad a la extinción de los mismos.

\section{AGRADECIMIENTOS}

Agradecemos a Don Rubén Estrella por su incondicional apoyo en el trabajo de campo, a Doña Rosita por su amabilidad y confianza que otorgo al primer autor durante su estancia en el rancho Hobonil, a todos los campesinos cazadores del municipio de Tzucacab, Yucatán que nos dieron la oportunidad de conocer y ser parte de esta actividad.

\section{LITERATURACITADA}

C.N.A. (Comisión Nacional del Agua). 2005. Registro de precipitación y temperatura mensuales de 2004- 2005. Mérida, Yucatán, México.

Duch, G. 1988. La conformación territorial del estado de Yucatán. Universidad Autónoma Chapingo. Edo. de México.

Escamilla, M., Sanvicente, M., Sosa, M. y C., Galindo-Leal. 2000. Habitat mosaic, 
wildlife availability, and hunting in the tropical forest of Calakmul, México. Conservation Biology, 14:1592-1601.

Flores, J. y E. Espejel. 1994. Tipos de vegetación de la Península de Yucatán. Etnoflora Yucatanense. Universidad Autónoma de Yucatán. México, Yucatán.

Gallegos, A., Bello, A. y A. Cruz .2004. Daños ocasionados por mamíferos terrestres en cultivos de maíz, en el Municipio de Tacotalpa, Tabasco. Pp.684-685, en: Memorias del VI Congreso Internacional sobre Manejo de Fauna Silvestre en la Amazonia y Latinoamérica. 5 - 10 Septiembre 2004, Iquitos-Perú.

Jorgenson, J.P. 1993. Gardens wildlife, and subsistence hunting by mayaindians in Quintana Roo, Mex-ico. PhD Thesis.University of Florida.

Jorgenson, J.P. 1995. Maya subsistence hunters in Quintana Roo, México.Oryx, 29: 49-57.

INEGI. 2010. Actividades primarias. $<$ http:// www.inegi.org.mx/sistemas/mexicocifras/ default.aspx?src $=487 \& e=31>$. Fecha de consulta: 20 de diciembre de 2011.

Kates, R. 2001. Sustainability Science. Science, 292:641-642.

León, P. 2006. Aprovechamiento de fauna silvestre en una comunidad aledaña a la Reserva de la Biosfera Los Petenes, Campeche, Tesis de Maestría en Ciencias en la especialidad de Ecología Humana, Centro de Investigación y de Estudios Avanzados del Instituto Politécnico Nacional, Yucatán, México.

Montiel S, L., Arias y F. Dickinson. 1999. La cacería tradicional en el norte de Yucatán: Una práctica comunitaria. Revista de Geografía Agrícola, 29: 43-52.

Martínez-Gallardo, R.y V., Sánchez-Cordero. 1997. Historia natural de algunas especies de mamiferos terrestres. Pp. 597-609., en:
Historia natural de los Tuxtlas. (Gonzales, E., R., Dirzo y R.C. Vogh, eds.) UNAM

March, I.J. 1995. Cacería de subsistencia, Usos locales e importancia cultural de la fauna silvestre en México. In: La fauna silvestre de México: necesidad de una nueva legislación. Asociación de Abogados de la Ciudad de México A.C. Fac. de Derecho, UNAM.

Municipios de México. 2002. Enciclopedia de los municipios de México: Yucatán. Instituto Nacional para el Federalismo y el Desarrollo Municipal - Gobierno del Estado de Yucatán 2001-2007.

Naranjo, E.J., Guerra, M., Bodmer, R. and J.E., Bolaños. 2004. Subsistence hunting by three ethnic groups of the Lacandon Forest, Mexico. Journal of Ethnobiology, 24:233-253.

Redford, K.H. y J., Robinson. 1997. Usos comerciales y de subsistencia de la vida silvestre en America Latina. Pp. 612, en: Usos y conservación de la vida silvestre neo-tropical. (Robinson, J. and K. Redford, eds.) Fondo de cultura Económica.

Redford, K.H. y J.G Robinson. 1987. The Game of choice: Patterns of indianand colonist hunting in the neotropic. American Antropologist, 89:650-667.

Reyna-Hurtado, R., G.W. Tanner. 2005. Habitat preferences of an ungulate community in Calakmul Forest, Campeche, Mexico.Biotropica, 37:676685.

Reyna-Hurtado, R., G.W. Tanner. 2007. Ungulate relative abundance in hunted and non-hunted sites in Calakmul Forest (Southern Mexico). Biodiversity and Conservation, 16:743-757.

Teran, S. y C. Rasmussen. 1992. La milpa bajo roza-tumba y quema en el Siglo XVI. Pp. 29-52, en: La modernización de la milpa 
en Yucatán: Utopía o realidad. (Zizumbo, V.D., C. Rasmussen, L.M. Arias y S. Terán, eds.) CICY-DANIDA-CP. Mérida, Yucatán. México.

Segovia C.A. 2001. La cacería de subsistencia en Tzucacab, Yucatán, Tesis de maestría. Universidad Autónoma de Yucatán, Mérida, Yucatán.

SEMARNAT. 2010. Norma Oficial Mexicana N O M - 059 - S E M A R N A T - 2010 , Protección ambiental-Especies nativas de México de flora y fauna silvestresCategorías de riesgo y especificaciones para su inclusión, exclusión o cambio-Lista de especies en riesgo.

Sowls L.K. 1997. Javelinas and other peccaries. Their biology and management. 2nd Ed, Texas A\&M University Press.

Vázquez, P.E., Méndez, R.M., Guiascón, O.G.R. y E.J., Naranjo. 2006. Uso medicinal de la fauna silvestre en los altos de Chiapas, México. Interciencia, 31:491499.

Vela, F. 2001. Un acto metodológico básico de la investigación social: la entrevista cualitativa. Pp. 61-131, en: Observar, escuchar y comprender sobre la tradición cualitativa en la investigación social. (Tarrés, M., coord.), Facultad Latinoamericana de Ciencias Sociales. Porrúa, México, D.F.

Vickers W.T. 1984.The faunal components of lowland South American hunting kills. Interciencia, 9:366-376.

\section{Nota:}

Espía: Técnica donde el cazador espera a su presa en un lugar determinado ya se arriba de un árbol o en una plataforma expresamente construida para cazar.
Batida: Técnica donde un grupo de cazadores revisan un área grande frecuentemente acompañados con perros con la intención de dirigir a la presa a un sitio determinado donde más cazadores esperan.

Lampareo: Técnica que consiste en cazar de noche con ayuda de linternas para distinguir los brillos de los ojos de los animales. 